Revista Perspectivas Online: Biológicas \& Saúde

Agosto de 2019, Vol.9, n 30, p.24-37

ISSN: 2236-8868 (Online)

DOI: $10.25242 / 886893020191743$

\title{
PERFIS SOCIOECONÔMICO, DEMOGRÁFICO, DE SAÚDE E ALIMENTAR DE IDOSOS DE ITUIUTABA/MG
}

\author{
Victor Antonio Ferreira Freire ${ }^{1}$ \& Luciana Karen Calábria ${ }^{1 *}$
}

\section{RESUMO}

FREIRE, V.A.F. \& CALÁBRIA, L.K. Perfis socioeconômico, demográfico, de saúde e alimentar de idosos de Ituiutaba/MG Perspectivas Online: Biológicas \& Saúde. v. 9, n 30, p.2437, 2019.

O estudo traçou e analisou conjuntamente os perfis socioeconômico, demográfico, de saúde e alimentar investigando suas relações e influências na saúde da população idosa atendida nas Unidades de Saúde do munícipio de Ituiutaba-MG. Para isso, 155 idosos, não institucionalizados, de ambos os sexos, responderam um questionário semiestruturado na forma de entrevista após assinatura do Termo de Consentimento Livre e Esclarecido. Prevaleceram idosos na faixa etária de 70-79 anos, do sexo feminino, e com baixas escolaridade e renda familiar. Quanto ao estado de saúde, os idosos referiram uso de prótese dentária e diagnóstico de diabetes e hipertensão. Em relação aos hábitos alimentares foi possível identificar uma parcela considerável de idosos que faz uso frequente de açúcar, sal, óleos e gorduras no preparo das refeições, bem como de cereais, com destaque nas frequências de 1 a 2 vezes na semana para o consumo de doces, realizando 2 a 3 refeições diárias, com preferência por alimentos de origem animal e vegetal. Ainda, a maioria dos idosos afirmou retirar o saleiro da mesa, e a pele e gordura aparente de carnes. Por outro lado, verificouse insuficiente ingestão de água, de prática de exercícios físicos e da leitura dos rótulos dos alimentos. Esses dados revelam consumo frequente de grupos alimentares específicos e comportamentos de risco associados ao desenvolvimento de doenças crônicas, as quais contribuem para o aumento de morbidade e redução da qualidade de vida dos idosos investigados.

Palavras-chave: Saúde do idoso; Dieta saudável; Perfil de saúde. 


\begin{abstract}
The study traced and analized the socioeconomic, demographic, health and to food profiles, and investigated their relationships and influences in the health of elderly population from Ituiutaba-MG. In this regard, 155 non-institutionalized elderly people, of both biological sex, answered a semi-structured questionnaire in the form of an interview after they signed the Free and Informed Consent Term. Elderly in the age of 70-79 years, female, and low schooling and family income prevailed. Regarding health status, the elderly reported use of dental prosthesis, and diagnosis of diabetes and hypertension. Regarding eating habits, it was possible to identify a considerable proportion

salt, oils and fats in the preparation of meals, as well as cereals, with emphasis on the frequencies of 1 to 2 times a week for the consumption of sweets, having 2 to 3 meals a day, with preference for food of animal and plant origin. Also, most elderly people said to remove the salt shaker from the table, and the apparent skin and fat from meat. On the other hand, there was insufficient water intake, physical exercise and reading of food labels. These data show frequent consumption of specific food groups and risk behaviors associated with the development of chronic diseases, which contribute to the increase of morbidity and reduction of quality of life of the elderly investigated.
\end{abstract} of elderly people that do frequent use sugar,

Keywords: Health of the Elderly; Health Diet; Health Profile.

\footnotetext{
${ }^{1}$ Universidade Federal de Uberlândia, Campus Pontal, Instituto de Ciências Exatas e Naturais do Pontal - Rua 20, 1600

- Tupã, Ituiutaba - MG, CEP: 38304-208 - Brasil.

(*)e-mail: 1kcalabria@ufu.br

Data de recebimento: 11/06/2019 Aceito para publicação: 22/08/2019
} 


\section{INTRODUÇÃO}

A população mundial, incluindo o Brasil, tem envelhecido num ritmo acelerado, sendo esse processo caracterizado pela redução nas taxas de fecundidade e de mortalidade, além do aumento da expectativa de vida. No entanto, o envelhecimento populacional acontece de maneira desigual, uma vez que é influenciado por questões socioeconômicas, políticas e geográficas (BRASIL, 2007). As estimativas apontam que em 2060, um quarto da população brasileira (25,5\%) terá 65 anos ou mais (58,2 milhões de idosos), o que demonstra um aumento vertiginoso da idade e do número de idosos, tendo como comparativo que as projeções indicavam um percentual de 9,2\% (19,2 milhões de idosos) para o ano de 2018 (AGÊNCIA IBGE NOTÍCIAS, 2018).

Desde a década de 60 tem ocorrido no Brasil uma mudança consistente no padrão de adoecimento da população (ARAÚJO, 2012), sendo as doenças crônicas não transmissíveis (DCNT) as mais prevalentes (SANTOS et al., 2014; PIMENTA et al., 2015; PAIVA et al., 2016; MELO et al., 2017a; SILVA et al., 2018), comprometendo o envelhecimento saudável e a expectativa de vida. Dentre as DCNT estão as doenças osteomusculares, hipertensão arterial sistêmica e doenças cardiovasculares, diabetes mellitus, doenças respiratórias crônicas, doenças cerebrovasculares, doenças orais e câncer (LAI; PARNELL; ORDOVAS, 2016).

De acordo com a Pesquisa Nacional de Saúde de 2013, Malta et al. (2017) revela que 45\% da população adulta brasileira têm diagnóstico de pelo menos uma doença crônica, e entre pessoas com 65 anos ou mais esse índice chega a 79,1\% (IBGE, 2010), o que torna as DCNT um forte agravante para saúde pública, uma vez que quase $50 \%$ da população pode atingir a velhice com sérios problemas de saúde decorrentes das suas manifestações clínicas, diminuindo de forma acentuada a qualidade de vida.

A alimentação inadequada é um dos determinantes na ocorrência e de óbitos por DCNT no Brasil (BRASIL, 2011). Entretanto, para os idosos a má alimentação tem impactos ainda maiores, sobretudo em decorrência das mudanças físicas, metabólicas e fisiológicas que ocorrem durante o envelhecimento e que exigem alterações nos padrões alimentares para suprir as suas necessidades nutricionais, uma vez que o declínio de suas funcionalidades afetam a ingestão e absorção de nutrientes (HAGEMEYER; REZENDE, 2013).

Pode-se ainda entender as alterações no estado nutricional do idoso, dentre as suas variadas formas, como o excesso, a deficiência ou a irregularidade alimentar, sendo um fator importante para o acometimento das doenças crônico-degenerativas que estão associadas à perda de autonomia, ao aumento da morbidade e da mortalidade (HAGEMEYER; REZENDE, 2013). Ainda, o estado nutricional do idoso pode ser afetado pelas condições ruins de saúde bucal, visto que a ausência de dentes prejudica a deglutição eficiente e a alimentação em geral, se fazendo necessário o uso de prótese dentária para correção das dificuldades de mastigação e deglutição (CARDOSO; BUJES, 2010).

Devido a esse fato, o levantamento e a análise de dados epidemiológicos referentes aos aspectos nutricionais e da saúde dos idosos são importantes, pois permitem reconhecer a condição e o estado de saúde da população em estudo, bem como a promoção de intervenções para melhoria desses padrões, divulgando iniciativas e comportamentos positivos voltados para melhoria da qualidade de vida. Sendo assim, este estudo teve como objetivo traçar e analisar conjuntamente os perfis socioeconômico, demográfico, de saúde e alimentar investigando suas relações e influências na saúde da população idosa atendida nas Unidades de Saúde do munícipio de Ituiutaba-MG. 


\section{METODOLOGIA}

\subsection{População alvo e questões éticas}

Trata-se de um estudo misto com delineamento transversal descritivo. A pesquisa foi conduzida no munícipio de Ituiutaba-MG, o qual apresentava uma populaçao aproximada de 97 mil habitantes em 2010, sendo 14 mil idosos. A estimativa populacional para o ano de 2018 era de 104 mil habitantes, revelando um aumento populacional de 6,6\% em relação ao ano de 2010 , o que provavelmente implica no aumento de idosos (IBGE, 2010).

O grupo populacional investigado incluiu 155 idosos (60 anos ou mais), de ambos os sexos (mulheres, $\mathrm{n}=89$; homens, $\mathrm{n}=66$ ), não institucionalizados, atendidos nas 12 Unidades de Saúde do munícipio, as quais estão distribuidas em 12 bairros, sendo eles, Alvorada, Camargo, Elândia, Independência, Jardim do Rosário, Natal, Novo Horizonte, Novo Tempo 2, Pirapitinga, Santa Maria, Setor Norte e Sol Nascente. Participaram do estudo os idosos que estavam aguardando atendimento médico na sala de espera ou que estavam participando das reuniões do HiperDia do Programa de Saúde da Família de cada Unidade, sendo assim consideradas as amostragens por conveniência e por julgamento, respectivamente.

Os idosos que apresentavam autonomia de resposta, aceitaram participar da pesquisa e assinaram o Termo de Consentimento Livre e Esclarecido aprovado pelos Comitês de Ética e Pesquisa da Universidade Federal de Uberlândia $\left(\mathrm{n}^{\circ}\right.$ 1.089.051) e da Universidade Federal de Juiz de Fora ( $\left.\mathrm{n}^{\mathrm{o}} 3.070 .463\right)$ foram incluídos no estudo. Por outro lado, foram excluídos os idosos que apresentaram incapacidade de responder a entrevista devido às dificuldades cognitivas e sensoriais que dificultaram a interpretação e articulação da resposta ou que desmonstraram algum viés de memória.

\subsection{Instrumento de coleta de dados e variáveis}

A coleta de dados foi realizada na forma de entrevista conduzida pelos membros do Grupo de Pesquisa de Atenção Preventiva e Educativa em Saúde do Idoso, os quais responderam diretamente no questionário semiestruturado (MELO et al., 2017a,b) constituído de perguntas referentes 1) às questões socioeconômicas e demográficas (logradouro, idade, sexo, situação conjugal, naturalidade, escolaridade, ocupação, renda e arranjo familiar), 2) aos hábitos alimentares (percepção de alimentação saudável; número de refeições; consumo de cereais, doces, óleo, gordura, sal e açúcar; preferência alimentar; consumo de carnes retirando pele e gordura; ingestão de água; retirada do saleiro; e leitura de rótulo dos alimentos) considerando os 10 passos para uma Alimentação Saudável para a Pessoa Idosa (BRASIL, 2009), 3) aos hábitos de vida (prática de atividade física, incluindo frequência semanal e modalidade) e 4) condição clínica em relação ao uso de prótese dentária e ao diagnóstico autorreferido para as doenças crônicas, como hipertensão arterial sistêmica (HAS), diabetes mellitus (DM) e doenças do aparelho osteomuscular (dores articulares, artrose e osteoporose).

\subsection{Análise dos dados}

Os dados foram tabulados utilizando o software Microsoft Office Excel 2010® e as análises das variáveis foram realizadas por meio de estatística descritiva considerando as frequências absoluta (n) e relativa (\%), média e desvio padrão.

\section{RESULTADOS}

O estudo foi conduzido com 155 idosos atendidos no sistema público de saúde de Ituiutaba- 
MG, sendo os indivíduos com idade média de 70,65 \pm 6,6 (mín: 60 anos e máx: 87 anos), com predominância da faixa etária de 70 a 79 anos $(49 \%)$ e de mulheres $(57,4 \%)$ (Tabela 1$)$.

A maior parte dos idosos declarou ter de 1 a 4 anos de estudo (47,1\%) e 29,7\% afirmaram ser analfabetos, incluindo analfabetos funcionais. Em relação à situação conjugal, 51,6\% afirmaram ter companheiro(a), apesar da maioria das mulheres relatar não possuir companheiro(a) (53,9\%). A maioria dos idosos declarou não morar sozinho, dos quais $36,1 \%$ vivem somente com companheiro(a) e 38,7\% declararam outro tipo de arranjo familiar (Tabela 1). A renda familiar mensal mais autorreferida foi menor que um mil reais $(44,5 \%)$, sendo a aposentadoria ou pensão $(85,8 \%)$ a principal fonte de renda (Tabela 1$)$.

Tabela 1: Perfil socioeconômico e demográfico dos idosos atendidos nas Unidades de Saúde, Ituiutaba-MG, 2018

\begin{tabular}{|c|c|c|c|}
\hline Variáveis & $\begin{array}{c}\text { Mulheres } \\
\text { n (\%) }\end{array}$ & $\begin{array}{c}\text { Homens } \\
\text { n }(\%)\end{array}$ & $\begin{array}{l}\text { Total } \\
\text { n }(\%)\end{array}$ \\
\hline \multicolumn{4}{|l|}{ Faixa etária (anos) } \\
\hline $60-69$ & $42(47,2)$ & $23(34,8)$ & $65(41,9)$ \\
\hline $70-79$ & $43(48,3)$ & $33(50,0)$ & $76(49,0)$ \\
\hline 80 ou mais & $4(4,5)$ & $10(15,2)$ & $14(9,1)$ \\
\hline \multicolumn{4}{|l|}{ Escolaridade } \\
\hline Analfabeto(a) & $14(15,7)$ & $5(7,6)$ & $19(12,3)$ \\
\hline Analfabeto(a) funcional & $16(18,0)$ & $11(16,7)$ & $27(17,4)$ \\
\hline 1 a 4 anos & $38(42,7)$ & $35(53,0)$ & $73(47,1)$ \\
\hline Mais de 4 anos & $21(23,6)$ & $15(22,7)$ & $36(23,2)$ \\
\hline \multicolumn{4}{|l|}{ Situação conjugal } \\
\hline Com companheiro $(a)$ & $41(46,1)$ & $39(59,1)$ & $80(51,6)$ \\
\hline Sem companheiro $(a)$ & $48(53,9)$ & $27(40,9)$ & $75(48,4)$ \\
\hline \multicolumn{4}{|l|}{ Arranjo familiar } \\
\hline Vive sozinho(a) & $21(23,6)$ & $18(27,3)$ & $39(25,2)$ \\
\hline Com companheiro $(a)$ & $31(34,8)$ & $25(37,9)$ & $56(36,1)$ \\
\hline Vive com filhos(as) com/sem companheiro(a) & $21(23,6)$ & $11(16,6)$ & $32(20,6)$ \\
\hline Vive com os netos(as) com/sem companheiro(a) & $16(18,0)$ & $12(18,2)$ & $28(18,1)$ \\
\hline \multicolumn{4}{|l|}{ Renda familiar mensal } \\
\hline Menos de $R \$ 1.000,00$ & $43(48,3)$ & $26(39,4)$ & $69(44,5)$ \\
\hline$R \$ 1.000$ a $2.000,00$ & $36(40,4)$ & $26(39,4)$ & $62(40,0)$ \\
\hline Mais de $R \$ 2.000,00$ & $9(10,1)$ & $14(21,2)$ & $23(14,8)$ \\
\hline Não responderam & $1(1,2)$ & $0(0,0)$ & $1(0,7)$ \\
\hline \multicolumn{4}{|l|}{ Fonte de renda } \\
\hline Aposentadoria ou pensionista & $72(80,9)$ & $61(92,4)$ & $133(85,8)$ \\
\hline Sem ocupação & $6(6,7)$ & $0(0,0)$ & $6(3,9)$ \\
\hline Com ocupação & $10(11,2)$ & $4(6,1)$ & $14(9,0)$ \\
\hline Não responderam & $1(1,2)$ & $1(1,5)$ & $2(1,3)$ \\
\hline
\end{tabular}

Mais da metade dos idosos referiu ter diagnóstico para doenças do aparelho circulatório, incluindo HAS $(82,6 \%)$ ou DM $(58,1 \%)$, sendo mais frequente nas mulheres (Tabela 2). Além disso, houve associação da prevalência da doença com menor tempo de estudo ( 0 a 4 anos) e com baixa renda familiar ( 0 até 2 mil reais) correspondendo à $80,5 \%$ e $87,4 \%$ para circulatório e $81,1 \%$ e $83,1 \%$ para DM dos idosos entrevistados com diagnóstico autorreferido, respectivamente. 
Em Ituiutaba-MG, 38,7\% dos idosos autorreferiram diagnóstico para o sistema osteomuscular, incluindo osteoporose, dor articular ou artrose. Mais da metade da população estudada apresentou algum tipo de doença crônica (Tabela 2), sendo que 52,9\% possuem diagnóstico autodeclarado para circulatório e DM, e 22,6\% para as três morbidades analisadas (Tabela 2). É importante ressaltar que possivelmente a prevalência de HAS e DM ocorreu em razão do grupo investigado ser atendido nas Unidades de Saúde e estar vinculado ao Programa HiperDia (Sistema de Cadastramento e Acompanhamento de Hipertensos e Diabéticos do Ministério da Saúde).

Tabela 2: Morbidades em idosos atendidos nas Unidades de Saúde, Ituiutaba-MG, 2018

\begin{tabular}{|c|c|c|c|}
\hline Diagnóstico autorreferido & $\begin{array}{l}\text { Mulheres } \\
\text { n }(\%)\end{array}$ & $\begin{array}{c}\text { Homens } \\
\text { n }(\%)\end{array}$ & $\begin{array}{l}\text { Total } \\
\text { n }(\%)\end{array}$ \\
\hline \multicolumn{4}{|l|}{ Diabetes mellitus } \\
\hline Sim & $54(60,7)$ & $36(54,5)$ & $90(58,1)$ \\
\hline Não & $35(39,3)$ & $30(45,5)$ & $65(41,9)$ \\
\hline \multicolumn{4}{|c|}{ Doenças do aparelho circulatório } \\
\hline Sim & $74(83,1)$ & $54(81,8)$ & $128(82,6)$ \\
\hline Não & $15(16,9)$ & $12(18,2)$ & $27(17,4)$ \\
\hline \multicolumn{4}{|c|}{ Doenças do sistema osteomuscular } \\
\hline Sim & $41(46,1)$ & $19(28,8)$ & $60(38,7)$ \\
\hline Não & $48(53,9)$ & $47(71,2)$ & $95(61,3)$ \\
\hline \multicolumn{4}{|c|}{ Doenças do aparelho circulatório e diabetes } \\
\hline Sim & $50(56,2)$ & $32(48,5)$ & $82(52,9)$ \\
\hline Não & $11(12,4)$ & $8(12,1)$ & $19(12,2)$ \\
\hline \multicolumn{4}{|c|}{$\begin{array}{l}\text { Diabetes, doenças do aparelho circulatório e do } \\
\text { sistema osteomuscular }\end{array}$} \\
\hline Sim & $24(27,0)$ & $11(16,7)$ & $35(22,6)$ \\
\hline Não & $5(5,6)$ & $7(10,6)$ & $12(7,7)$ \\
\hline
\end{tabular}

Ao serem questionados sobre a sua própria percepção de alimentação saudável, 76,4\% das mulheres idosas e $89,4 \%$ dos homens idosos afirmaram ter uma boa alimentação (Tabela 3), relatando preferência para os alimentos de origem vegetal e animal $(88,4 \%)$ em oposição aos industrializados (Tabela 3).

Em relação ao consumo diário de sal, açúcar, gorduras e óleo, 51,6\% dos idosos afirmaram realizá-lo com frequência no preparo dos alimentos, com prevalência entre os homens $(62,1 \%)$, apesar de $44,5 \%$ do total investigado afirmar ter diminuído o seu uso. Quanto à disposição do saleiro na mesa, $84,5 \%$ dos idosos entrevistados afirmaram retirá-lo durante as refeições (Tabela 3 ).

Conforme observado na Tabela 3, houve também maior prevalência no consumo de doces nas frequências de 1 a 2 vezes/semana (75,5\%), consumo diário frequente de cereais $(53,5 \%)$ e retirada de pele e gordura aparente das carnes brancas e vermelhas antes de seu preparo ou consumo $(63,9 \%)$. Ainda, $49,7 \%$ relataram fazer de 2 a 3 refeições, com destaque para os homens, e 42,6\% de 4 a 5, com prevalência para as mulheres (Tabela 3).

Além disso, 43,2\% dos idosos afirmaram ingerir dois ou mais litros diários de água, sendo as mulheres a minoria. Por outro lado, a maioria dos idosos afirmou fazer o uso de prótese dentária $(63,9 \%)$, destacando o público feminino $(69,7 \%)$, e relatou nunca ter lido os rótulos dos alimentos antes de comprá-los e/ou consumi-los (60\%). 
Tabela 3: Hábitos alimentares de idosos atendidos nas Unidades de Saúde, Ituiutaba-MG, 2018

\begin{tabular}{|c|c|c|c|}
\hline Hábitos alimentares & $\begin{array}{c}\text { Mulheres } \\
\text { n }(\%)\end{array}$ & $\begin{array}{c}\text { Homens } \\
\text { n }(\%)\end{array}$ & $\begin{array}{l}\text { Total } \\
\text { n }(\%)\end{array}$ \\
\hline Alimentação saudável & $68(76,4)$ & $59(89,4)$ & $127(81,9)$ \\
\hline \multicolumn{4}{|l|}{ Preferência alimentar } \\
\hline Origem vegetal e animal & $81(91,0)$ & $56(84,8)$ & $137(88,4)$ \\
\hline Industrializados & $8(9,0)$ & $10(15,2)$ & $18(11,6)$ \\
\hline \multicolumn{4}{|c|}{ Consumo diário de açúcar/gorduras/sal/óleo } \\
\hline Com frequência & $39(43,8)$ & $41(62,1)$ & $80(51,6)$ \\
\hline Tem diminuído & $47(52,8)$ & $22(33,3)$ & $69(44,5)$ \\
\hline Não utiliza & $3(3,4)$ & $3(4,6)$ & $6(3,9)$ \\
\hline \multicolumn{4}{|c|}{ Disponibilidade de saleiro na mesa } \\
\hline Sim & $14(15,7)$ & $9(13,6)$ & $23(14,8)$ \\
\hline Não & $74(83,1)$ & $57(86,4)$ & $131(84,5)$ \\
\hline Não responderam & $1(1,2)$ & $0(0,0)$ & $1(0,7)$ \\
\hline \multicolumn{4}{|l|}{ Consumo de doces } \\
\hline 1-2 vezes/semana & $68(76,3)$ & $49(74,2)$ & $117(75,5)$ \\
\hline 3-4 vezes/semana & $7(7,9)$ & $8(12,1)$ & $15(9,7)$ \\
\hline 5-6 vezes/semana & $7(7,9)$ & $3(4,6)$ & $10(6,4)$ \\
\hline Todos os dias & $7(7,9)$ & $6(9,1)$ & $13(8,4)$ \\
\hline \multicolumn{4}{|l|}{ Consumo diário de cereais } \\
\hline Sempre & $45(50,6)$ & $38(57,6)$ & $83(53,5)$ \\
\hline Às vezes & $34(38,2)$ & $24(36,4)$ & $58(37,4)$ \\
\hline Não consome & $10(11,2)$ & $4(6,0)$ & $14(9,1)$ \\
\hline \multicolumn{4}{|c|}{ Consumo diário de carnes sem pele/gordura } \\
\hline Sempre & $64(71,9)$ & $35(53,0)$ & $99(63,9)$ \\
\hline Às vezes & $11(12,4)$ & $13(19,7)$ & $24(15,5)$ \\
\hline Não retira & $14(15,7)$ & $18(27,3)$ & $32(20,6)$ \\
\hline \multicolumn{4}{|c|}{ Número de refeições e lanches diários } \\
\hline 1 & $0(0,0)$ & $1(1,6)$ & $1(0,6)$ \\
\hline $2-3$ & $40(44,9)$ & $37(56,1)$ & $77(49,7)$ \\
\hline $4-5$ & $44(49,4)$ & $22(33,3)$ & $66(42,6)$ \\
\hline 6 ou mais & $5(5,7)$ & $6(9,0)$ & $11(7,1)$ \\
\hline \multicolumn{4}{|l|}{ Ingestão diária de água } \\
\hline Menor que 500 mililitros & $9(10,1)$ & $5(7,6)$ & $14(9,1)$ \\
\hline 500 mililitros a 1 litro & $33(37,1)$ & $12(18,2)$ & $45(29,0)$ \\
\hline 1 litro a 1,5 litros & $16(18,0)$ & $13(19,7)$ & $29(18,7)$ \\
\hline Mais que 2 litros & $31(34,8)$ & $36(54,5)$ & $67(43,2)$ \\
\hline
\end{tabular}

A atividade física foi relatada por 52,2\% dos idosos; entretanto, apenas 50,6\% dos idosos investigados afirmaram praticar 150 minutos ou mais de atividade física durante a semana, preferencialmente as mulheres (53,8\%). A tabela 4 também apresenta os dados referentes às modalidades praticadas, sendo a caminhada $(66,8 \%)$ e a ginástica $(12,4 \%)$ as mais citadas (Tabela 4). 
Tabela 4: Prática de atividade física por idosos atendidos nas Unidades de Saúde, ItuiutabaMG, 2018

\begin{tabular}{lccc}
\hline Variáveis & $\begin{array}{c}\text { Mulheres } \\
\mathbf{n}(\%)\end{array}$ & $\begin{array}{c}\text { Homens } \\
\mathbf{n}(\%)\end{array}$ & $\begin{array}{c}\text { Total } \\
\mathbf{n}(\%)\end{array}$ \\
\hline Prática de atividade física & & & \\
Sim & $39(43,8)$ & $42(63,6)$ & $81(52,2)$ \\
Não & $50(56,2)$ & $24(36,4)$ & $74(47,8)$ \\
Frequência semanal & & & \\
Menos de 150 minutos & $18(46,2)$ & $22(52,4)$ & $40(49,4)$ \\
150 ou mais minutos & $21(53,8)$ & $20(47,6)$ & $41(50,6)$ \\
Modalidade & & & \\
Caminhada & $22(56,4)$ & $32(76,2)$ & $54(66,8)$ \\
Alongamento & $3(7,7)$ & $0(0,0)$ & $3(3,7)$ \\
Musculação & $2(5,1)$ & $2(4,8)$ & $4(4,9)$ \\
Ciclismo & $0(0,0)$ & $4(9,5)$ & $4(4,9)$ \\
Dança & $3(7,7)$ & $1(2,4)$ & $4(4,9)$ \\
Pilates & $1(2,6)$ & $0(0,0)$ & $1(1,2)$ \\
Ginástica & $7(17,9)$ & $3(7,1)$ & $10(12,4)$ \\
Não responderam & $1(2,6)$ & $0(0,0)$ & $1(1,2)$ \\
\hline
\end{tabular}

\section{DISCUSSÃO}

Os resultados desse estudo corroboram com os diagnósticos feitos por diversos autores, nos quais predomina o sexo feminino, apesar de revelarem a faixa etária de 60 a 69 anos como a mais prevalente (SANTOS et al., 2014; PIMENTA et al., 2015; PAIVA et al., 2016; MATIAS; MATIAS; ALENCAR, 2016; MELO et al., 2017b; SILVA et al., 2018) e o baixo grau de escolaridade (SANTOS et al., 2014; MATIAS; MATIAS; ALENCAR, 2016; PAIVA et al., 2016; SILVA et al., 2018), confirmando o envelhecimento progressivo da população atendida nas Unidades de Saúde do município de Ituiutaba-MG.

Os dados socieconômicos da população idosa investigada em 2018 revela uma diminuição da sua renda familiar quando comparada com o levantamento realizado por Melo et al. (2017b) em 2015 no mesmo munícipio.

Quando analisado o diagnóstico autorreferido de doenças do aparelho circulatório (+6,8\%) e do DM (+20,6\%), observa-se aumento nas frequências de 2018 comparadas com as de 2015 (Melo et al., 2017a). Na investigação realizada pelo Vigitel (BRASIL, 2018) por inquérito telefônico nas capitais brasileiras foi encontrada predominância na autodeclaração de HAS pelas mulheres. Entretanto, no que se refere ao DM não houve associação significativa entre os sexos e a frequência de diagnóstico aumentou com a idade e a menor escolaridade (0 a 8 anos de estudo) para ambas as doenças (circulatório e DM). Cabe destacar que em nosso estudo houve prevalência de HAS e DM entre as mulheres, considerando o sexo e o diagnóstico autorreferido.

De acordo com a Organização Mundial de Saúde (WHO, 2018a), as DCNT, incluindo HAS e DM, são um problema de saúde responsável por $71 \%$ dos óbitos mundiais, e os idosos em decorrência do envelhecimento são numericamente mais afetados, sofrendo com seus agravamentos por longos períodos, uma vez que essas doenças resultam no acometimento de outras enfermidades, limitações fisiológicas e físicas, e até mesmo de incapacidades (WHO, 2005; BRASIL, 2011). O 
atual cenário das DCNT no mundo revela elevada taxa de mortalidade prematura em indivíduos mais jovens, apesar de altos índices ainda serem detectados para pessoas com 70 anos ou mais (BENNETT et al., 2018). Ao mesmo tempo em que as estimativas atuais demostram a gravidade desse quadro, retratam também um cenário emergencial para o futuro, uma vez que a velhice poderá ser marcada pela redução da qualidade de vida.

Semelhante ao contexto mundial, os óbitos no Brasil em decorrência de DCNT chegaram a $74 \%$ em 2016, com prevalência das doenças cardiovasculares (28\%), cânceres (18\%), respiratórias (6\%) e do DM (5\%). Entre os fatores que justificam essas mortes estão os fisiológicos, como a hipertensão, hiperglicemia, hipercolesterolemia e sobrepeso, os quais são resultado dos fatores comportamentais, como o uso exagerado de álcool e de tabaco, inatividade física e má alimentação (WHO, 2018b). Entretanto, estudos retratam que as dietas não saudáveis são responsáveis pela maior taxa de doenças globais apresentando um risco maior para morbidade e a mortalidade do que a combinação do uso de bebidas alcoólicas, drogas, tabaco ou do sexo sem proteção (GLOBAL PANEL, 2016; WILLETT et al., 2019).

Se tratando do perfil alimentar dos idosos investigados, a autopercepção positiva de alimentação saudável se mostra representativa. Por outro lado, os idosos justificaram não ter alimentação saudável devido à baixa renda familiar, à alimentação fora do lar e às restrições dietéticas prescritas pelo médico em função do tratamento de alguma doença crônica. Como pontuado por Fazzio (2012), os aspectos sociais, culturais e singulares do idoso influenciam a sua prática alimentar, bem como interferem na sua escolha, consumo e acesso aos alimentos.

O comportamento alimentar da população idosa em Ituiutaba-MG comparado ao longo de três anos (MELO et al., 2017b) revela aumento no consumo de sal, açúcar, gorduras e óleo, embora atualmente as mulheres tenham se destacado na diminuição do uso em relação aos homens. Seguindo as recomendações do "Guia alimentar para a população brasileira" (BRASIL, 2014), a qualidade nutricional de um prato depende dos alimentos que o compõe, bem como de seu preparo, sendo necessário equilíbrio quanto à utilização de sal, açúcar, gorduras e óleo.

A retirada do saleiro da mesa é uma iniciativa significativa para a prevenção e auxílio do tratamento da HAS e é uma prática alimentar dos idosos investigados. Nesse sentido, a manutenção desse comportamento, como também de uma alimentação saudável e sustentável em sua totalidade, equilíbrio da ingestão e do gasto calórico, prática de exercícios físicos e interrupção do uso de tabaco previnem complicações que podem estar ou não associadas à HAS, como a morte súbita, acidentes vasculares encefálicos, infarto agudo do miocárdio, insuficiência cardíaca, doença arterial periférica e doença renal (MALACHIAS et al., 2016).

Ao comparar os dados sobre o consumo de doces, cerais e massas obtidos em 2018 com os resultados de Melo et al. (2017b) levantados em 2015, observa-se aumento no consumo diário de alimentos restritos para indivíduos diagnosticados com pré-diabetes ou DM tipo 1 e 2 , sendo $+11,7 \%$ para doces e $+9,2 \%$ para cereais e massas, como milho, pães, massas em geral, batata e raízes. A estratégia nutricional é um passo importante na prevenção e no tratamento do DM, uma vez que a partir dela é possível atingir o equilíbrio metabólico fundamental para a qualidade de vida e para a redução das complicações e chances de desenvolver doenças cardiovasculares. A alimentação saudável nesse aspecto contribui para o equilíbrio energético, além da manutenção do peso corporal, dos níveis pressóricos e lipídicos, os quais são essenciais para o controle da glicemia em diabéticos. Em relação aos hábitos alimentares, a prevenção do DM envolve uma estratégia nutricional que inclua o consumo de cerais integrais, frutas, legumes e verduras, além da ingestão reduzida de carnes vermelhas, levando também em consideração a individualidade fenotípica (OLIVEIRA et al., 2017). Isso porque os alimentos de origem animal são boas fontes de proteínas,

Persp. online: biol. \& saúde, Campos dos Goytacazes, 30 (9),24- 37, 2019 
vitaminas e minerais, mas são pobres em fibras e ricos em gorduras saturadas, características que favorecem o risco de sobrepeso e de doenças crônicas (BRASIL, 2014).

Considerando as refeições diárias, o hábito de realizar pelo menos café da manhã, almoço, jantar e dois lanches saudáveis por dia, sem pular refeições importantes, é o primeiro passo para uma alimentação saudável e equilibrada de acordo com os "Dez passos para uma alimentação saudável para pessoas idosas" (BRASIL, 2009). Outro hábito que contribui para um padrão saudável de alimentação é a leitura de rótulos dos alimentos, uma prática pouco realizada pelos idosos investigados, mas que auxilia na diferenciação dos alimentos ultraprocessados dos processados e é uma realidade em outras regiões do Brasil (ver LINDEMANN et al., 2016).

O "Guia alimentar para população brasileira" não traz uma recomendação específica e quantitativa para ingestão de água, uma vez que a ingestão diária pode variar com a idade, o peso, a prática e frequência de atividade física, além de levar em consideração o clima e a temperatura do ambiente local (BRASIL, 2014). A atenção especial à ingestão de água durante a velhice se faz necessária, visto que com o envelhecimento, alterações na função do sistema nervoso associadas ou não à redução da sensibilidade dos osmorreceptores levam à menor sensação de sede (CAMPOS et al., 2000). Além disso, a utilização de medicamentos com ação diurética e laxante, dificuldade motora ou dependência física que possa limitar o acesso à água, elevadas temperaturas e a prática de exercícios físicos favorecem à perda de água e desidratação (MARUCCI; FERREIRA, 2008). Na literatura também é possível encontrar variação quanto à recomendação diária; por exemplo, o Caderno de Atenção Básica (BRASIL, 2007) orienta a ingestão de pelo menos dois litros (6 a 8 copos) diários de água, preferencialmente, no intervalo das refeições, enquanto Marucci e Ferreira (2008) indicam a ingestão diária de 30 a $35 \mathrm{~mL}$ por quilograma de peso.

Estudos demostram a prevalência na utilização de próteses dentárias por idosos (BERTOTTI et al., 2015; PIMENTA et al., 2015; FARIA et al., 2016) indiferentemente do sexo. De acordo com Nico et al. (2016) os idosos brasileiros são os que mais perderam dentes nos últimos anos $(41,5 \%)$. Azevedo et al. (2017) destacam a existência de diferenças regionais quanto ao uso e necessidade de prótese dentária, sendo relacionadas ao contexto socioeconômico, demográfico e a utilização de serviços odontológicos. Nesse sentido, ficam claros a gravidade e o grau da perda de dentes, e até mesmo de edentulismo na população estudada e em escala nacional (AZEVEDO et al., 2017). O acúmulo e a progressão de afecções bucais juntamente com a desigualdade de acesso e o uso de serviços odontológicos podem justificar o elevado grau de edentulismo na população idosa (BARROS; BERTOLDI, 2002; MOREIRA et al., 2005). Além disso, a falsa crença de que com o avanço da idade, a perda de dentes e o uso de próteses são imprescindíveis pode acabar construindo uma sensação de normalidade e ao mesmo tempo ausência de hábitos preventivos à saúde bucal (MARTINS; BARRETO; PORDEUS, 2009), o que na verdade é resultado da carência de prevenção, informação e higienização adequada (SIMÕES; CARVALHO, 2011). Sendo assim, as orientações dietéticas e a integração de atendimento médico e dos serviços odontológicos são vitais para a promoção de saúde dos idosos (BRASIL, 2008).

Como parte do processo de senescência, há também a perda de massa muscular e o aumento no percentual de gordura (SILVA et al., 2016). Por outro lado, a prática de atividade física tem um importante papel protetor para as doenças crônicas, obesidade e as limitações funcionais, evitando a dependência do idoso às atividades diárias no ambiente familiar ou social, além de prover benefícios aos sistemas sensório-motores que melhoram o equilíbrio (ARANTES, 2016; FREITAS; KOPILER; CAMPOS, 2013). A Organização Mundial da Saúde (WHO, 2010) recomenda 150 minutos de prática regular de exercício físico moderado semanalmente, sendo que para os idosos são indicados exercícios físicos que busquem estabelecer o fortalecimento muscular, equilíbrio e a resistência física, como os treinos de musculação, hidroginástica e dança (ARANTES, 2016). 
A atividade física traz benefícios à saúde física, mental e social. Em geral, diversos eventos que ocorrem ao longo da vida do idoso podem cooperar para o seu declínio cognitivo, como a depressão, hábitos comportamentais, questões sociais e psicológicas; no entanto, uma vida ativa é capaz de reestabelecer a qualidade de vida social e metal, uma vez que possibilita ao idoso reintegrar a sua independência e participação social (WHO, 2005). A Organização Mundial da Saúde (WHO, 2005) destaca de maneira generalizada a sustentação de três pilares para promoção do envelhecimento ativo: a participação, a segurança e a saúde. Somado a isso, a participação do idoso na sociedade e no contexto familiar contribui de maneira positiva para o seu bem-estar e, consequentemente, para o estado de saúde.

Questões de ordem social influenciam de forma acentuada na prática alimentar, inclusive atribuindo para a socialização como aspecto integrante e benéfico para o comportamento alimentar, uma vez que os isolamentos nos papéis sociais e familiares podem ser danosos à saúde, pela possiblidade de gerar descuido com a alimentação, bem como a elevação do consumo de alimentos industrializados e redução no consumo de alimentos in natura (SANTOS; RIBEIRO, 2011).

\section{CONCLUSÕES}

A partir deste estudo foi possível observar que por mais que os idosos de Ituiutaba-MG atendidos nas Unidades de Saúde sejam preferencialmente mulheres, de baixa renda e com baixa escolaridade, apresentam preferência no consumo de alimentos saudáveis, de origem animal e vegetal como, frutas, verduras, legumes, cereais, carnes, leite e seus derivados, reconhecendo a sua importância para uma boa prática alimentar, ainda que a não leitura dos rótulos seja uma realidade na vida desses idosos.

A prevalência de doenças crônicas, principalmente de HAS e DM, é justificada parcialmente pelo comportamento assumido por esses idosos, apesar de não termos realizado o levantamento de quanto tempo eles convivem com o diagnóstico, mas destacando o consumo diário de açúcar, gordura, sal, óleo e cereais, e a baixa adesão na prática de exercício físico. Além disso, outros aspectos identificados também influenciam os hábitos alimentares e a saúde destes idosos, como a baixa ingestão de água, o número reduzido de refeições diárias e o frequente uso de prótese dentária, os quais precisam ser orientados por meio de ações de educação preventiva em saúde para melhoria da qualidade de vida geral dessa população.

\section{REFERÊNCIAS}

AGÊNCIA IBGE NOTÍCIAS. Projeção da População 2018: número de habitantes do país deve parar de crescer em 2047. 2018. Disponível em: https://agenciadenoticias.ibge.gov.br/agencia-salade-imprensa/2013-agencia-de-noticias/releases/21837-projecao-da-populacao-2018-numero-dehabitantes-do-pais-deve-parar-de-crescer-em-2047. Acesso em: 11 mar. 2019.

ARANTES, R. C. Diretrizes para a gestão do envelhecimento ativo e com qualidade de vida para idosos brasileiros. In: FARIA, L.; CALÁBRIA, L.K.; ALVES, W.A. Envelhecimento: um olhar interdisciplinar. São Paulo: Hucitec, 2016. p. 188-214.

ARAÚJO, J. D. Polarização epidemiológica no Brasil. Epidemiologia e Serviços de Saúde, v. 21, n. 4, p. 533-538, 2012.

AZEVEDO, J. S. et al. Uso e necessidade de prótese dentária em idosos brasileiros segundo a Pesquisa Nacional de Saúde Bucal (SBBrasil 2010): prevalências e fatores associados. Cadernos de Saúde Pública, v. 33, n.8, p. 1-12, 2017.

BARROS, A. J. D.; BERTOLDI, A. D. Desigualdades na utilização e no acesso a serviços 
odontológicos: uma avaliação em nível nacional. Ciência e Saúde Coletiva, v. 7, n. 4, p. 709-717, 2002.

BENNETT, J. E. et al. NCD Countdown 2030: worldwide trends in non-communicable disease mortality and progress towards Sustainable Development Goal target 3.4. The Lancet, v. 392, n. 10152, p.1072-1088, 2018.

BERTOTTI, M. E. Z. et al. Autopercepção da saúde bucal de idosos em interface com doenças crônicas e uso de medicações. Arquivos Médicos dos Hospitais e da Faculdade de Ciências Médicas da Santa de Casa São Paulo, v. 60, n. 2, p. 54-60, 2015.

BRASIL. Ministério da Saúde. Envelhecimento e saúde da pessoa idosa. Brasília, DF, 2007. Disponível em: http://bvsms.saude.gov.br/bvs/publicacoes/abcad19.pdf. Acesso em: 11 dez. 2018.

BRASIL. Ministério da Saúde. Saúde Bucal. 1. ed. Brasília, DF, 2008. Disponível em: http://bvsms.saude.gov.br/bvs/publicacoes/saude_bucal.pdf. Acesso em: 11 dez. 2018.

BRASIL. Ministério da Saúde. Alimentação saudável para a pessoa idosa: um manual para profissionais de saúde. Brasília, DF, 2009. Disponível em: http://bvsms.saude.gov.br/bvs/publicacoes/alimentacao_saudavel_idosa_profissionais_saude.pdf. Acesso em: 13 mar. 2019.

BRASIL. Ministério da Saúde. Plano de ações estratégicas para o enfrentamento das doenças crônicas não transmissíveis (DCNT) no Brasil 2011-2022. Brasília, DF, 2011. Disponível em: http://bvsms.saude.gov.br/bvs/publicacoes/plano_acoes_enfrent_dent_2011.pdf. Acesso em: 13 mar. 2019.

BRASIL. Ministério da Saúde. Guia alimentar para a população brasileira. 2.ed. Brasília, DF, $2014 . \quad$ Disponível em: http://bvsms.saude.gov.br/bvs/publicacoes/guia_alimentar_populacao_brasileira_2ed.pdf. Acesso em: 13 mar. 2019.

BRASIL. Ministério da Saúde. Vigitel Brasil 2017: vigilância de fatores de risco e proteção para doenças crônicas por inquérito telefônico. Brasília, DF, 2018. Disponível em: http://bvsms.saude.gov.br/bvs/publicacoes/vigitel_brasil_2017_vigilancia_fatores_riscos.pdf. Acesso em: 13 mar. 2019.

CAMPOS, M. T. F. S.; MONTEIRO, J. B. R.; ORNELAS, A. P. R. C. Fatores que afetam o consumo alimentar e a nutrição do idoso. Revista de Nutrição, v. 13, n. 3, p. 157-165, 2000.

CARDOSO, M. C. A. F.; BUJES, R. V. A saúde bucal e as funções da mastigação e deglutição nos idosos. Estudos Interdisciplinares sobre o Envelhecimento, v. 15, n. 1, p. 53-67, 2010.

FARIA, L. et al. Atenção preventiva e educativa em saúde do idoso: uma proposta de integração de saberes e práticas. Estudos Interdisciplinares sobre o Envelhecimento, v. 21, n. 1, p. 35-54, 2016.

FAZZIO, D. M. G. Envelhecimento e qualidade de vida: uma abordagem nutricional e alimentar. Revista de Divulgação Científica Sena Aires, v. 1, n. 1, p. 76-88, 2012.

FREITAS, E. V.; KOPILER, D. A.; CAMPOS, A. L. Atividade física. In: FREITAS, E. V. et al. Tratado de geriatria e gerontologia. 3. ed. Rio de Janeiro: Guanabara Koogan, 2013. p. 874-887.

GLOBAL PANEL ON AGRICULTURE AND FOOD SYSTEMS FOR NUTRITION (GLOBAL PANEL). Food systems and diets: facing the challenges of the 21st century. London, 2016. Disponível em: http://glopan.org/sites/default/files/ForesightReport.pdf. Acesso em: 11 mar. 2019.

HAGEMEYER, V.; REZENDE, C. H. A. Nutrição e Envelhecimento. In: FREITAS, E. V. et al. Tratado de geriatria e gerontologia. 3. ed. Rio de Janeiro: Guanabara Koogan, 2013. p. 1459- 
1477.

INSTITUTO BRASILEIRO DE GEOGRAFIA E ESTATÍSTICA (IBGE). Um panorama da Saúde no Brasil: acesso e utilização dos serviços, condições de saúde e fatores de risco e proteção à saúde (PNAD 2008). Rio de Janeiro, RJ, 2010. Disponível em: http://bvsms.saude.gov.br/bvs/publicacoes/pnad_panorama_saude_brasil.pdf. Acesso em: 11 mar. 2019.

INSTITUTO BRASILEIRO DE GEOGRAFIA E ESTATÍSTICA (IBGE). IBGE cidades. 2010. Disponível em: https://cidades.ibge.gov.br/brasil/mg/ituiutaba/panorama. Acesso em: 13 ago. 2019.

LAI, C.; PARNELL, L. D.; ORDOVAS, J. M. Genetic mechanisms of aging. In: FILLIT, H. M.; ROCKWOOD, K.; YOUNG, J. Brocklehurst's textbook of geriatric medicine and gerontology. 8 ed. Philadelphia: Elsevier, 2016. p. 43-46.

LINDEMANN, I. L. et al. Leitura de rótulos alimentares entre usuários da atenção básica e fatores associados. Cadernos Saúde Coletiva, v. 24, n. 4, p. 478-486, 2016.

MALACHIAS, M. V. B. et al. $7^{\text {a }}$ Diretriz brasileira de hipertensão arterial. Arquivos Brasileiros de Cardiologia, v. 107, n. 3, supl. 3, p. 1-83, 2016.

MALTA, D. C. et al. Doenças crônicas não transmissíveis e a utilização de serviços de saúde: análise da Pesquisa Nacional de Saúde no Brasil. Revista de Saúde Publica, v. 51, Supl. 1:4s, 2017.

MARTINS, A. M. E. B. L.; BARRETO, S. M.; PORDEUS, I. A. Auto-avaliação de saúde bucal em idosos: análise com base em modelo multidimensional. Cadernos de Saúde Pública, v. 25, n. 2, p.421-435, 2009.

MARUCCI, M. F. N.; FERREIRA, L. S. Ações preventivas na terceira idade. In: FILHO, W. J.; GORZONI, M. L. Geriatria e Gerontologia: o que todos devem saber. São Paulo: Roca; 2008. p. 63-83.

MATIAS, C. O. F.; MATIAS, C. O. F.; ALENCAR, B. R. Qualidade de vida em idosos portadores de Diabetes Mellitus Tipo 2 atendidos em Unidades Básicas de Saúde de Montes Claros/MG. Revista Brasileira de Qualidade de Vida, v. 8, n. 2, p. 119-129, 2016.

MELO, J. V. et al. Perfil de saúde dos idosos atendidos nas Unidades Básicas de Saúde da Família (UBSF) em Ituiutaba, Minas Gerais. Revista de Atenção à Saúde, v. 15, n. 53, p. 66-75, 2017a.

MELO, J. V. et al. Hábitos alimentares dos idosos atendidos nos Programas de Saúde da Família (PSF) em Ituiutaba-MG. Revista de Medicina e Saúde de Brasília, v. 6, n. 2, p. 154-166, 2017 b.

MOREIRA, R. S. et al. A saúde bucal do idoso brasileiro: revisão sistemática sobre o quadro epidemiológico e acesso aos serviços de saúde bucal. Cadernos de Saúde Pública, v. 21, n. 6, p. $1665-1675,2005$.

NICO, L. S. et al. Saúde bucal autorreferida da população adulta brasileira: resultados da Pesquisa Nacional de Saúde 2013. Ciência e Saúde Coletiva, v. 21, n. 2, p. 389-398, 2016.

OLIVEIRA, J. E. P.; MONTENEGRO JUNIOR, R. M.; VENCIO, S. Diretrizes da Sociedade Brasileira de Diabetes 2017-2018. São Paulo: Editora Clannad, 2017. Disponível em: https://www.diabetes.org.br/profissionais/images/2017/diretrizes/diretrizes-sbd-2017-2018.pdf.

Acesso em: 13 mar. 2019.

PAIVA, M. H. P. et al. Fatores associados à qualidade de vida de idosos comunitários da macrorregião do Triângulo do Sul, Minas Gerais, Brasil. Ciência Saúde Coletiva, v. 21, n. 11, p. 3347-3356, 2016.

PIMENTA, F. B. et al. Fatores associados a doenças crônicas em idosos atendidos pela Estratégia

Persp. online: biol. \& saúde, Campos dos Goytacazes, 30 (9),24- 37, 2019 
de Saúde da Família. Ciência Saúde Coletiva, v. 20, n. 8, p. 2489-2498, 2015.

SANTOS, A. S. et al. Perfil de saúde e qualidade de vida de idosos de Santa Juliana-MG. Enfermagem em Foco, v. 5, n. 3/4, p. 61-64, 2014.

SANTOS, G. D.; RIBEIRO, S. M. L. Aspectos afetivos relacionados ao comportamento alimentar dos idosos frequentadores de um Centro de Convivência. Revista Brasileira de Geriatria e Gerontologia, v. 14, n. 2, p. 319-328, 2011.

SILVA, C. L. A. et al. Mudanças no estado nutricional e suas condições de risco para o idoso. In: FARIA, L.; CALÁBRIA, L.K.; ALVES, W.A. Envelhecimento: um olhar interdisciplinar. São Paulo: Hucitec, 2016. p. 215-243.

SILVA, P. A. B. et al. Perfil sociodemográfico e clínico de idosos acompanhados por equipes de Saúde da Família sob a perspectiva do gênero. Revista de Pesquisa: Cuidado é Fundamental Online, v. 10, n. 1, p. 97-105, 2018.

SIMÕES, A. C. A.; CARVALHO, D. M. A realidade da saúde bucal do idoso no Sudeste brasileiro. Ciência e Saúde Coletiva, v. 16, n. 6, p. 2975-2982, 2011.

WORLD HEALTH ORGANIZATION (WHO). Envelhecimento ativo: uma política de saúde. Brasília: Organização Pan-Americana da Saúde, 2005. Disponível em: http://bvsms.saude.gov.br/bvs/publicacoes/envelhecimento_ativo.pdf. Acesso em: 13 mar. 2019.

WORLD HEALTH ORGANIZATION (WHO). Global recommendations on physical activity for health. Genebra, $2010 . \quad$ Disponível em: http://apps.who.int/iris/bitstream/handle/10665/44399/9789241599979_eng.pdf;jsessionid=CAF4D 956836E90EA7D53BA54B8EC7197?sequence=1. Acesso em: 07 nov. 2018.

WORLD HEALTH ORGANIZATION (WHO). World health statistics 2018: monitoring health for the SDGs, sustainable development goals. Genebra, 2018a. Disponível em: https://apps.who.int/iris/bitstream/handle/10665/272596/9789241565585-eng.pdf?ua=1. Acesso em: 13 mar. 2019.

WORLD HEALTH ORGANIZATION (WHO). Noncommunicable diseases country profiles 2018. Geneva, 2018b. Disponível em: https://www.who.int/nmh/publications/ncd-profiles-2018/en/. Acesso em: 13 mar. 2019.

WILLETT, W. et al. Food in the Anthropocene: the EAT - Lancet Commission on healthy diets from sustainable food systems. The Lancet, v. 393, n. 10170, p. 447-492, 2019. 